\title{
High Resolution Ultrasonographic Evaluation of Salivary Glands Lesions with Clinico-Pathological Correlation in Government Medical College, Thrissur, Kerala
}

\author{
Arunkumar Shankar Mukesh ${ }^{1}$, Sinni Kareepadath Vivekanandan² \\ ${ }^{1}$ Department of Radiology, Lourdes Hospital, Ernakulam, Kerala, India. ${ }^{2}$ Department of Radiology, \\ Government Medical College, Thrissur, Kerala, India.
}

\section{ABSTRACT}

\section{BACKGROUND}

Salivary gland swelling presents with a variety of neoplastic and non-neoplastic conditions. Predicting the nature of a swelling on clinical grounds alone is rarely possible hence, further assessment with accurate imaging is a necessity. Ultrasonography is a diagnostic tool that is widely available, relatively inexpensive, non-invasive, and easily reproducible. This study was undertaken to assess the role of ultrasonography as a diagnostic aid in various salivary gland swellings.

\section{METHODS}

Cross sectional study was conducted for consecutive patients who came to the Radiology Department, Govt. Medical College, Thrissur, from January 2014 to January 2015. Ultrasound for clinically suspected salivary gland lesions was done. USG scanning was done by Mindray DC - 08 high resolution real time multiplanar diagnostic Ultrasound System with linear array high frequency 6 to $10 \mathrm{MHZ}$ transducer having scan head of $60 \mathrm{~mm}$.

\section{RESULTS}

Out of the 67 patients who were included in the study, 34 cases were identified correctly by ultrasound examination and confirmed by clinical follow up or by histopathology. Fifteen cases were A/C sialadenitis which were confirmed clinically, 11 cases were salivary gland abscess which were confirmed clinicopathologically. Eight cases were sialolithiasis which were confirmed by histopathology. Thirtythree cases were neoplastic of which 6 were malignant and 27 were benign which were confirmed by histopathology. Of the 6 malignant lesions which were confirmed by histopathology, ultrasonography correctly identified 5 cases. All these 27 benign cases were diagnosed as benign through sonography also. However out of the 18 pleomorphic adenoma cases, 15 were correctly diagnosed by USG. Out of the 9 cases of Warthin's tumour confirmed by histopathology, USG correctly identified 7 cases.

\section{CONCLUSIONS}

Ultrasonography is a valuable supplement in the diagnosis of salivary gland lesions and to distinguish neoplastic/ non-neoplastic lesion so that further management can be decided accordingly.

\section{KEY WORDS}

Pathology, Salivary Gland Neoplasm, Ultrasonography
Corresponding Author:

Dr. Sinni Kareepadath Vivekanandan, Department of Radiology, Government Medical College,

Thrissur, Kerala, India.

E-mail:drsinnikv@gmail.com

DOI: $10.14260 / j e m d s / 2021 / 796$

How to Cite This Article:

Mukesh AS, Vivekanandan SK. High resolution ultrasonographic evaluation of salivary glands lesions with clinicopathological correlation in government medical college, Thrissur, Kerala. J Evolution Med Dent Sci 2021;10(45):39413946, DOI: 10.14260/jemds/2021/796

Submission 26-09-2021, Peer Review 03-10-2021, Acceptance 20-12-2021, Published 28-12-2021.

Copyright (C) 2021 Arunkumar Shankar Mukesh et al. This is an open access article distributed under Creative Commons Attribution License [Attribution 4.0 International (CC BY 4.0)] 


\section{BACKGROUND}

Salivary glands are important structures which secrete saliva, that take part in food digestion process. In addition to saliva carrying some digestive enzymes, it also takes part in the body defence system through its concentration of antibodies. The accumulation of bacteria on intra oral structures is prevented by the constant flow of saliva in the mouth which in turn reduces the chances of infection. Speech and mastication are made easy and comfortable through the lubrication effect of saliva. However, salivary glands often develop diseases and conditions that can affect their basic functions. Metabolic disorders, infections (fungal, bacterial, and viral), neoplastic conditions (both benign and malignant), cyst and occasionally trauma can affect the function of a single or several glands. Such conditions often cause discomfort or pain resulting in physical and psychological incapacitation. Salivary gland diseases rarely present with any symptoms during the early stages except for acute infections which are usually painful. ${ }^{1}$ Because of this, patients stay with their diseases for a long time before reporting to hospital. Majority of them present with advanced disease that may complicate the management due to the huge size or metastasis. Most of the salivary gland disorders present as non-specific tumours without any unique features. Because of the complicated anatomy of the maxillofacial region, it requires sophisticated imaging techniques like $\mathrm{x}$ rays, ultrasonography, CT or / and MRI to be able to accurately see the extension of the tumour. This is necessary in planning of the definitive treatment.

Unfortunately, most of these investigations are not available in lower-level health facilities. The definitive diagnosis is possible only through histological investigations. Although the treatment of salivary gland tumours is basically surgical, the approach differs according to their behaviour and stage of the disease when patients report for health care. Salivary gland diseases / conditions affect all age groups with some of them showing a sex predilection. The distribution of lesions among the different salivary glands has been reported to show a difference in occurrence between whites and blacks. For non-African populations, more than $80 \%$ of salivary gland neoplasms have been found to involve the parotid gland ${ }^{1}$. Much less were seen in the submandibular gland, minor salivary glands, or the sublingual gland. ${ }^{1}$

Management of bacterial infections of salivary glands often calls for an aggressive use of antibiotics. Rarely is incision and drainage required. Viral infections (like mumps) usually need symptomatic treatment and reassurance. Since salivary gland tumours do not respond to chemotherapy and are virtually radioresistant, surgery remains the only treatment of choice. A variety of neoplastic and nonneoplastic conditions may present with salivary gland swelling. It is rarely possible to predict the nature of a swelling on clinical grounds alone and accurate imaging is necessary for further assessment. ${ }^{2}$

Although computed tomography and magnetic resonance imaging are valuable diagnostic imaging aids, both are expensive and not universally available. ${ }^{3}$ CT scan carries the risk of radiation exposure in addition. Ultrasonography is an alternative diagnostic tool that is widely available, relatively inexpensive, non-invasive, and easily reproducible. ${ }^{4}$
The salivary glands are superficial structures and are readily amenable to high resolution ultrasound examination. Ultrasound evaluation is quick and non-invasive. Ultrasonography can differentiate possible benign from malignant neoplasms. ${ }^{2}$ It can demonstrate features of inflammation or abscess formation. USG can differentiate whether a palpable lesion over salivary gland region arises within the salivary gland or is peri glandular in location. USG is helpful in differentiating cystic from solid lesions; aid in guiding the exact site of Fine Needle Aspiration Cytology (FNAC) in suspected salivary gland lesions. ${ }^{5}$ When combined with colour Doppler imaging, it helps in assessing the vascularity and nature of the lesion (malignant lesions of salivary glands are highly vascular as compared to their benign counterparts). RI and PI values of greater than 0.7 and 1.2, respectively, coupled with high PSV (greater than $44.3 \mathrm{~cm}$ / s), ill-defined margins, and nodal involvement with central vascularity are highly indicative of malignant salivary gland lesion. ${ }^{6}$ USG helps in differentiating intra parotid lymph nodes from true intra-parenchymal lesions, identifying calcifications / diffuse lesions and detecting major ductal dilatation with intraductal calculi. ${ }^{7}$

Therefore, high resolution ultrasonography is valuable in identifying various pathologies of salivary glands. With this background in mind, this study was undertaken to assess the role of ultrasonography as a diagnostic aid in various salivary gland swellings.

\section{METHODS}

A cross sectional study was done. The subjects were selected from regular out / in patient visiting the Department of Radiology, Government Medical College, Thrissur, for ultrasonographic evaluation of clinically suspected salivary gland lesion between January 2014 and January 2015 (12 months). All patients who were referred for USG evaluation of clinically suspected salivary gland swelling were included. Those who were unwilling to give a valid consent for the study, patients who had undergone surgery for the same lesion previously and those patients who were not followed up in our hospital were excluded.

After getting valid consent from the study subjects, detailed history was taken, clinical examination findings and clinical diagnosis were filled in the proforma. The patients were then subjected to ultrasonographic examination. USG scanning was done using MINDRAY high resolution, real time multiplanar diagnostic ultrasound system with a linear array, high frequency (triple frequency) 6 to $10 \mathrm{MHz}$ transducer, having scan head of $60 \mathrm{~mm}$. The USG findings were entered into the proforma and the ultrasonographic diagnosis was reached. The patients were then subjected to histopathological examination for confirmation of diagnosis which is considered as the gold standard. For the confirmatory diagnosis of acute sialadenitis and salivary gland abscess, clinical follow up (response to medical treatment) and findings on incision \& drainage were taken as diagnostic gold standard respectively. 


\section{Statistical Analysis}

Data collected from each individual was entered into an Excel Sheet after coding of variables. Appropriate analysis was done with the help of Epi-info software. Sensitivity, specificity, predictive value and diagnostic accuracy for ultrasonography in the diagnosis of salivary gland swellings was calculated.

\section{RESULTS}

\section{Distribution of Patients by Age and Sex}

67 patients were included in the study. Of them $42(62.69 \%)$ were males and 25 (37.31\%) were females. Out of the 67 patients, most of the patients were in the age group of $31-40$ years (23.88\%). The age ranged between 9 and 78 years with a mean of 39.9 years. Only 2 patients were below the age of 10 years and no patients were above 80 years.

\section{Distribution of Salivary Gland Diseases by Age and Sex}

Pleomorphic adenoma was the commonest lesion encountered in the study affecting 18 (26.85\%) patients of whom $14(77.75 \%)$ were males and 4 (22.25\%) were females. Majority of patients with pleomorphic adenoma belonged to the 41 - 50 years age group i.e., 7 patients $(38.8$ $\%)$; followed by 5 patients (27.7\%) in the $31-40$ years age group.

Warthin's tumour was observed in $9(13.4 \%)$ patients of whom 7 (77.7 \%) were males and $2(22.3 \%)$ were females. Majority of patients with Warthin's tumour belonged to the 61 - 70 years age group i.e., 4 patients (44.4\%). Malignant salivary gland tumours were observed in $6(8.9 \%)$ patients comprised of $4(66.6 \%)$ males and $2(33.4 \%)$ females. Majority of patients affected by malignant salivary gland tumours were aged above 60 years - 4 patients $(66.6 \%)$. The commonest observed malignant salivary gland tumour was mucoepidermoid carcinoma in 5 (83.3 \%) patients. The remaining one was confirmed to be adenoid cystic carcinoma on histopathology.

Among the non-neoplastic lesions, acute sialadenitis was the commonest lesion affecting $15(22.4 \%)$ patients followed by salivary gland abscess $11(16.4 \%)$ patients and sialolithiasis $8(11.9 \%)$ patients. Majority of patients with sialadenitis belonged to the $10-30$ years age group - 12 patients $(80 \%)$. Majority of patients with sialolithiasis were aged between 10 and 25 years - 4 patients (50\%). Majority of patients with salivary gland abscess belonged to the $30-40$ years age group - 7 patients $(63.6 \%)$.

\section{Distribution of Salivary Gland Diseases by Anatomical Site \\ Major salivary glands were affected in all cases of the study. Amongst the major salivary gland, parotid gland was involved in $44(65.6 \%)$ patients followed by submandibular gland in $23(34.4 \%)$ patients. No minor salivary gland lesions were detected in the study. In the study, non-neoplastic lesions were observed in 34 (50.75\%) patients. Among the non- neoplastic lesions sialedenitis and sialolithiasis constitutes 23}

(67.6\%) patients. Most cases of sialedinitis were observed to involve parotid gland (66.6\%) whereas sialolithiasis was common in submandibular gland (87.5\%).

In the study neoplastic lesions were observed in $33(49.2$ $\%$ cases. Among them benign neoplasms were common (81.8\%) of which pleomorphic adenoma was the commonest benign neoplasm encountered (66.6\%). Most of pleomorphic adenoma was observed to involve parotid gland (66.6\%).

The remaining cases involved submandibular gland. All cases of Warthin' s tumour was observed to involve parotid gland.

Mucoepidermoid carcinoma was observed to involve parotid gland in $60 \%$ cases followed by submandibular gland in $40 \%$ cases. Only one case of adenoid cystic carcinoma was found in the study and it involved the parotid gland.

\section{Ultrasonographic Features}

Acute Sialadeinitis

In all cases of acute sialedinitis, the salivary glands were diffusely enlarged. Echo pattern was hypoechoic in $66.6 \%$ cases and rest showed heterogenous echo texture. All of the cases showed increased internal vascularity and enlarged regional lymph nodes.

\section{Salivary Gland Abscess}

Salivary gland abscess showed anechoic echo pattern in 63.6 $\%$ of cases or hypoechoic echo pattern in $36.4 \%$ of cases. Boarders were ill defined in $72 \%$ cases with central avascular area and debris in all cases.

\section{Sialolithiasis}

All cases of sialolithiasis showed hyperechoic foci with distal posterior acoustic shadowing, which represented calculus.

\section{Pleomorphic Adenoma}

Pleomorphic adenoma was found to be well defined in all cases. Lesion was lobular in $66.6 \%$ of cases, hypoechoic in 72 $\%$ of cases and hypo vascular in $88 \%$ of cases. Calcifications were demonstrated in $22 \%$ of cases.

\section{Warthin's Tumour}

All cases were well defined lesions. Most of the Warthin's tumours were oval in $54.4 \%$ cases, hypoechoic in $64 \%$ of cases and few were hyper vascularized (44.4\%). Calcifications were seen in $33 \%$ cases.

\section{Malignant Neoplasms}

Mucoepidermoid carcinoma showed an irregular shape in $66.66 \%$ of cases, irregular borders in $60 \%$ of cases, blurred margins in $64.5 \%$ of cases, and a hypoechoic inhomogeneous parenchyma in $60 \%$ of cases. The lesion was hyper vascular in $61.6 \%$ of cases and showed solid cystic components in $62.6 \%$ of cases.

\begin{tabular}{|ccc|}
\hline Age Group & Frequency & Percentage \\
$0-10$ & 2 & $2.99 \%$ \\
$11-20$ & 13 & $19.40 \%$ \\
$21-30$ & 5 & $7.46 \%$ \\
$31-40$ & 16 & $23.88 \%$ \\
$41-50$ & 13 & $19.40 \%$ \\
$51-60$ & 6 & $8.96 \%$ \\
$61-70$ & 8 & $11.94 \%$ \\
$71-80$ & 4 & $5.97 \%$ \\
Total & $\mathbf{6 7}$ & $\mathbf{1 0 0 . 0 0} \%$ \\
\hline Table 1. Age Wise Distribution of Patients \\
\hline
\end{tabular}




\begin{tabular}{|ccc|}
\hline Gender & Frequency & Percent \\
Male & 42 & $62.69 \%$ \\
Female & 25 & $37.31 \%$ \\
Total & $\mathbf{6 7}$ & $\mathbf{1 0 0 . 0 0} \%$ \\
\hline Table 2. Gender Wise Distribution of Patients \\
\hline
\end{tabular}

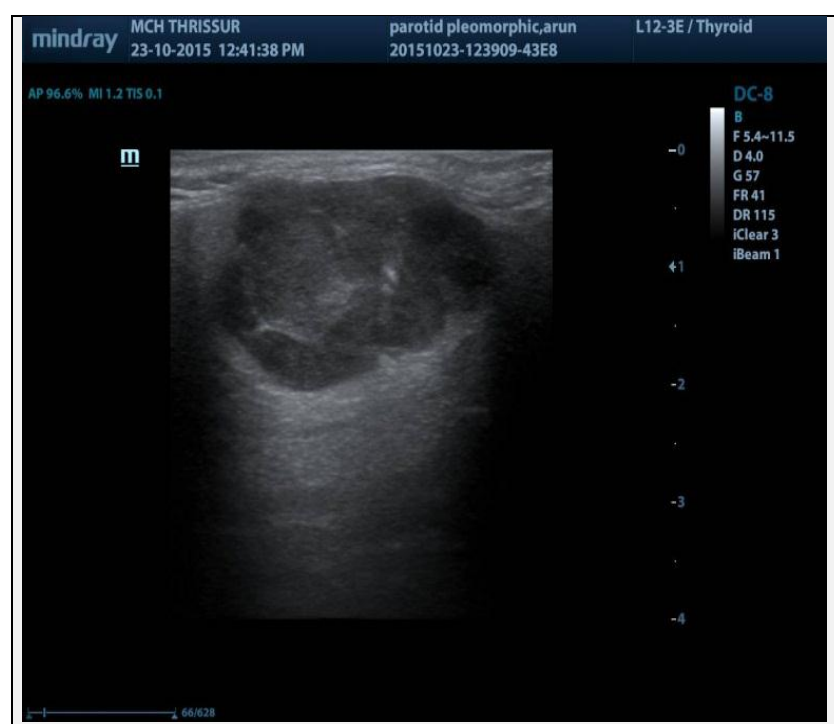

Figure 1. A Case of Parotid Pleomorphic Adenoma. The Lesion is Hypoechoic and Lobulated with Distinct Borders and Posterior Aco ustic Enhancement

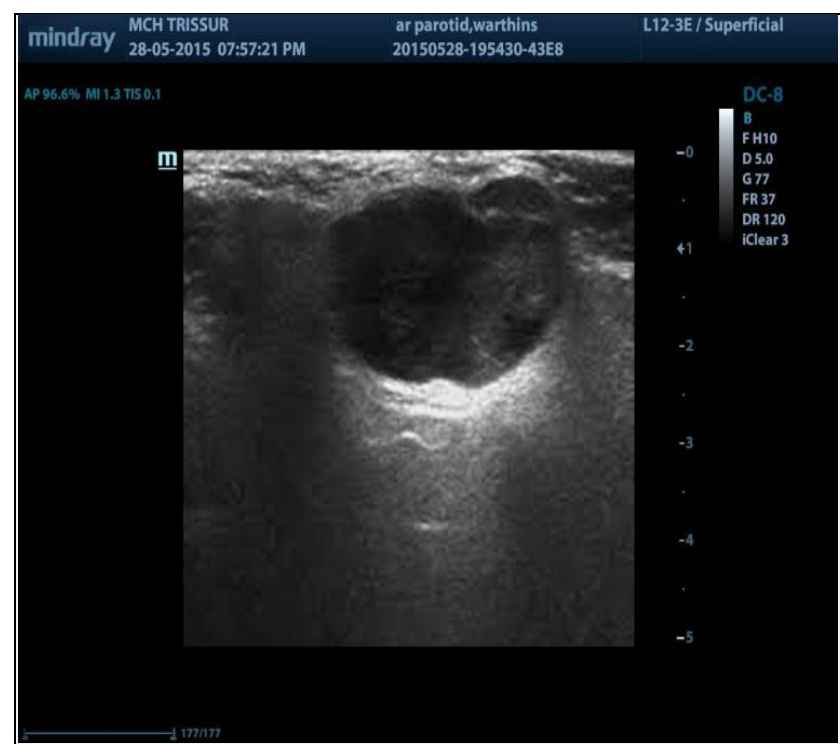

Figure 2. A Case of Warthin's Tumour. The Lesion Appear Hypoechoic, Lobulated, with Well-defined Boarders. Small Anechoic Areas Can Be Seen

\begin{tabular}{|ccc|}
\hline Abscess & 11 & $16.42 \%$ \\
Adenoid cystic carcinoma & 1 & $1.49 \%$ \\
Acute sialadenitis & 15 & $22.39 \%$ \\
Mucoepidermoid carcinoma & 5 & $7.46 \%$ \\
Pleomorphic adenoma & 18 & $26.87 \%$ \\
Sialolithiasis & 8 & $11.94 \%$ \\
Warthin's tumour & 9 & $13.43 \%$ \\
Total & $\mathbf{6 7}$ & $\mathbf{1 0 0 . 0 0} \%$ \\
\hline Table 3. Frequency of Lesions in Final Diagnosis \\
\hline
\end{tabular}

\begin{tabular}{|cccc|}
\hline Diagnosis & Correct & Incorrect & $\begin{array}{c}\text { \% of Correctly } \\
\text { Diagnosed Cases }\end{array}$ \\
Non neoplastic & 34 & 0 & 100.0 \\
Benign neoplasms & 22 & 5 & 81.4 \\
Malignant neoplasms & 5 & 1 & 83.3 \\
Total & $\mathbf{6 1}$ & $\mathbf{6}$ & $\mathbf{9 1 . 0 4}$ \\
\hline Table 4. Correlation of USG and Clinicopathological Finding \\
\hline
\end{tabular}

\begin{tabular}{|c|cccc|}
\hline Salivary Gland Lesion & Sensitivity & Specificity & PPV & NPV \\
Pleomorphic adenoma & $83.33 \%$ & $95.92 \%$ & $88.24 \%$ & $94.00 \%$ \\
Warthin's tumour & $77.78 \%$ & $93.10 \%$ & $63.64 \%$ & $96.43 \%$ \\
Mucoepidermoid carcinoma & $80.00 \%$ & $94.2 \%$ & $90.5 \%$ & $98.41 \%$ \\
ACUTE sialadenitis & $100.00 \%$ & $100.00 \%$ & $100.00 \%$ & $100.00 \%$ \\
Sialolithiasis & $100.00 \%$ & $100.00 \%$ & $100.00 \%$ & $100.00 \%$ \\
Salivary gland abscess & $100.00 \%$ & $100.00 \%$ & $100.00 \%$ & $100.00 \%$ \\
\hline Table 5. Sensitivity, Specificity, PPV and NPV of Ultrasonography \\
\hline
\end{tabular}

\section{DISCUSSION}

In this study it was found that salivary gland diseases mostly occurred in patients younger than 40 years with the highest frequency in the 4 th decade. It showed that there were more males affected than females at a ratio of 1.7:1. Both neoplastic and non-neoplastic lesions commonly involve the parotid gland. Malignant tumours were more common in males. Salivary stones were most often located in the submandibular gland. Lustmann J et al. ${ }^{8}$ studied 245 patients with sialolithiasis, treated during a period of 20 years. They found that submandibular gland was involved in 231 patients, the parotid gland in 11 patients and the sublingual gland only in 1 patient. Among neoplastic lesions, benign neoplasms were more common in which pleomorphic adenoma was the commonest tumour and among inflammatory conditions sialadenitis was the commonest lesion. The most commonly encountered lesion among the malignant tumours was mucoepidermoid carcinoma. Li LJ et al. ${ }^{9}$ studied 3461 cases of salivary gland tumours retrospectively between year 1955 2002 and they concluded that primary tumours mostly occur in parotid, and pleomorphic adenoma was the most common benign tumour followed by Warthin's tumour. The most common malignant tumour was mucoepidermoid followed by adenoid cystic carcinoma. In this study, all cases of acute sialadenitis showed diffusely enlarged salivary glands. Echo pattern was hypoechoic in majority of cases and rest showed heterogenous echotexture. All cases showed increased internal vascularity and enlarged regional lymph nodes. Ching AS et al. ${ }^{10}$ studied 256 cases over a period of 3 years and concluded cases of acute sialadenitis show diffusely enlarged salivary glands with hypoechoic texture in majority of cases. Majority of cases showed increased internal vascularity and enlarged regional lymph nodes. In this study abscesses were hypoechoic or anechoic lesions with unclear borders. The avascular area or moving debris indicated central liquefaction.

Nozaki $\mathrm{H}$ et al. ${ }^{11}$ studied six children with recurrent parotitis by ultrasound. Of the 12 parotid glands thus observed, 5 were swollen and 7 were not enlarged. Multiple round hypoechoic areas measuring $2-4 \mathrm{~mm}$ in diameter were seen in all 5 enlarged parotid glands and in 5 nonenlarged glands. The hypoechoic areas were suggested to represent both peripheral sialectasis and surrounding lymphocytic infiltration. Ultrasonography was proposed to be useful for the diagnosis and follow-up of recurrent parotitis in childhood. In this study sialolithiasis was characterised by the presence of hyperechoic foci with distal posterior acoustic shadowing, which represented calculus. Similar finding was reported by Traxler $\mathrm{M}$ et al.12 Their study included 47 patients over a period of 2 years. In the study pleomorphic adenomas were hypoechoic, well defined, 
lobulated tumours with posterior acoustic enhancement and a very few cases contained calcifications.

Vascularization in pleomorphic adenomas was often poor or absent (even when the sensitive power Doppler mode is used). Bialek EJ et al.13 studied 88 patients and showed ultrasound was able to differentiate between benign and malignant lesions with $96 \%$ accuracy. There was up to $84 \%$ accuracy to predict that the tumour detected was pleomorphic adenoma. The demonstration of histopathologic heterogeneity of pleomorphic adenomas in 16 primary tumours [73\%] was possible through high resolution probes and harmonic imaging.

About $95 \%(21 / 22)$ of the primary pleomorphic adenomas had 5 or fewer vessels which were detected in the whole lesion. Calcifications were rarely seen but the lesions were mostly hypoechoic, lobulated with posterior acoustic enhancement. The study showed that Warthin's tumours were well-defined, oval, hypoechoic and hypervascularization was seen in a few. Kim J et al. ${ }^{14}$ retrospectively evaluated the sonograms of Warthin's tumours of the parotid gland that had been confirmed by histopathologic examination of surgically excised specimens from patients over a 2-year period.

The tumours were categorized into 2 groups on the basis of size ( $<5 \mathrm{~cm}$ vs $\geq 5 \mathrm{~cm}$ in the largest diameter) and their echogenicity, shape, margin, and internal structure were recorded. The analysis included 18 patients (16 men and 2 women) whose mean age was 57 years with a range of 29-82 years. There was only 1 tumour in 17 patients while one patient had 2 tumours ( 1 in each parotid gland).

The findings from ultrasonography showed that all 19 tumours were hypoechoic while the affected parotid glands had normal parenchyma. Ovoid shape, well-defined margins and multiple, small, irregular, sponge-like anechoic areas were seen in most of the tumours. There was a higher proportion of cystic content in tumours that were $\geq 5 \mathrm{~cm}$ in diameter compared with smaller lesions and a portion of cases were composed almost entirely of cystic material. It was concluded that there is a distinction in the sonographic appearance of a Warthin's tumour. The mass is well-defined round or ovoid and hypoechoic which contains microcystic anechoic areas. Although for confirmation of the diagnosis, sonographically guided fine-needle aspiration biopsy may be required, these sonographic features constitute useful criteria in the preoperative diagnosis of Warthin's tumour in the parotid gland.

The study showed a hypoechoic homogeneous parenchyma with irregular shape, border and blurred margins in majority of mucoepidermoid carcinoma. The lesions were hyper vascular in most of the cases and showed solid-cystic components. Bradley et al.15 prospectively assessed fifty-six patients with salivary gland lesions using ultrasound imaging with colour flow and power Doppler. The peak systolic velocity (PSV), the pulsatility index (PI) and resistive index (RI) were determined. Histology was correlated with the real time ultrasound morphology and the Doppler information. No internal colour flow or power Doppler changes could be detected in 18 out of the 56 patients. Most of the lesions showed irregular shape, irregular borders, blurred margins, and heterogenous parenchyma. The lesions were hyper vascular in most of the cases and showed solid-cystic components.

Elevation of the PSV was seen in several cases $(>50 \mathrm{~cm}$ per s) however, no statistical correlation with malignancy was found. The sensitivity and specificity of ultrasonography when compared to clinical outcome were found to be $100 \%$ in cases of sialolithiasis, acute sialadenitis and salivary gland abscess. For pleomorphic adenoma, Warthin's tumour and mucoepidermoid carcinoma ultrasonography showed a sensitivity of $80.00 \%, 77.78 \%$ and $83.33 \%$ respectively. For the same lesions USG showed a specificity of $95.92 \%, 93.10$ $\%$ and $94.2 \%$ respectively. N. Gritzmann et al. ${ }^{16}$ in their retrospective study of 850 patients over 5 years found that tumours of the salivary glands can be visualized with high sensitivity (> $90 \%$ ).

Sriskandan N et al. (2010) ${ }^{17}$ evaluated 220 patients who presented with palpable parotid lesions over a period of 11 years and they found that 201 patients had focal lesions: 29 carcinomas, 21 lymphomata and 151 benign lesions (including 69 pleomorphic adenomas and 54 Warthin's tumours); 19 patients did not have focal lesions. Out of 201 focal lesions, 25 were indeterminate in the initial ultrasound report. The sensitivity, specificity and diagnostic accuracy for malignancy was $91 \%, 93 \%$ and $93 \%$, respectively in the remaining 176 lesions. Kamble RC et al.18 (2013) in their study found out that ultrasonography is valuable and useful method for diagnosis of salivary gland diseases. From the study, it is clear that there was $100 \%$ congruency between final diagnosis and ultrasonographic diagnosis in all the cases of non-neoplastic lesions. For benign and malignant neoplasms, a congruency of $81 \%$ and $83 \%$ were noted respectively. Overall diagnostic accuracy of ultrasonography on salivary gland lesions was found to be $98.64 \%$ in the present study. There was also a significant association between ultrasonographic diagnosis and histopathological diagnosis ( $\mathrm{p}$ value $<0.001$ ).

\section{CONCLUSIONS}

Ultrasonography showed significantly high sensitivity and specificity in diagnosing non-neoplastic lesions and thus, ultrasonography evaluation of salivary gland lesions helps in the decision making of surgical intervention. Therefore, ultrasonography is a valuable supplement in the diagnosis of salivary gland lesions and to distinguish neoplastic / nonneoplastic lesion so that further management can be decided accordingly.

Data sharing statement provided by the authors is available with the full text of this article at jemds.com.

Financial or other competing interests: None.

Disclosure forms provided by the authors are available with the full text of this article at jemds.com.

\section{REFERENCES}

[1] Kerr AG; Scott-Brown's otolaryngology, 6 $6^{\text {th }}$ edn, Vol. 5, Oxford: Butterworth-Heinemann International 1997:19/1-21/15 
[2] Howlett DC High resolution ultrasound assessment of the parotid gland. Br J Radiol 2003;76(904):271-7.

[3] Peleg M, Heyman Z, Ardekian L, et al. The use of ultrasonography as a diagnostic tool for superficial fascial space infection. J Oral Maxillofac Surg 1998;56(10):1129-31.

[4] Chodosh PL, Silbey R, Oen KT. Diagnostic use of ultrasound in diseases of the head and neck. The Laryngoscope 1980;90(5 Pt 1):814-21.

[5] Cho HW, Kim J, Choi J, et al. Sonographically guided fineneedle aspiration biopsy of major salivary gland masses: a review of 245 cases. AJR Am J Roentgenol 2011;196(5):1160-3.

[6] El-Khateeb SM, Abou-Khalaf AE, Farid MM, et al. A prospective study of three diagnostic sonographic methods in differentiation between benign and malignant salivary gland tumours. Dentomaxillofac Radiol 2011;40(8):476-85.

[7] Bialek EJ, Jakubowski W, Zajkowski P, et al. US of the major salivary glands: anatomy and spatial relationships, pathologic conditions, and pitfalls. Radiographics 2006;26(3):745-63.

[8] Lustmann J, Regev E, Melamed Y. Sialolithiasis: a survey on 245 patients and a review of the literature. Int J Oral Maxillofac Surg 1990;19(3):135-8.

[9] Li LJ, Li Y, Wen YM, et al. Clinical analysis of salivary gland tumor cases in West China in past 50 years. Oral Oncology 2008;44(2):187-92.

[10] Ching AS, Ahuja AT, King AD, et al. Comparison of the sonographic features of acalculous and calculous submandibular sialadenitis. J Clin Ultrasound 2001;29(6):332-8.

[11] Nozaki H, Harasawa A, Hara H. et al. Ultrasonographic features of recurrent parotitis in childhood. Pediatr Radiol 1994;24(2):98-100.

[12] Traxler M, Schurawitzki H, Ulm C, et al. Sonography of nonneoplastic disorders of the salivary glands. Int J Oral Maxillofac Surg 1992;21(6):360-3.

[13] Bialek EJ, Jakubowski W, Karpinska G. Role of ultrasonography in diagnosis and differentiation of pleomorphic adenomas: work in progress. Arch Otolaryngol Head Neck Surg 2003;129(9):929-33.

[14] Kim J, Kim EK, Park CS, et al. Characteristic sonographic findings of Warthin's tumour in the parotid gland. J Clin Ultrasound 2004;32(2):78-81.

[15] Bradley PJ, Eisele DW. Salivary Gland Neoplasms in Children and Adolescents. Adv Otorhinolaryngol 2016;78:175-81.

[16] Gritzmann N, Macheiner P. Lipoma in the parotid gland: typical US and CT morphology. Ultraschall Med 2003;24(3):195-6.

[17] Sriskandan N, Hannah A, Howlett DC. A study to evaluate the accuracy of ultrasound in the diagnosis of parotid lumps and to review the sonographic features of parotid lesions - results in 220 patients. Clin Radiol 2010;65(5):366-72.

[18] Kamble RC, Joshi AN, Mestry PJ. Ultrasound characterization of salivary lesions. Int J Otorhinolaryngol Clin 2013;5(4):16-29. 